\title{
Necessary and Sufficient Conditions for a Class Positive Local Martingale
}

\author{
Chuanzhong Chen, Saisai Yang \\ Department of Mathematics, Hainan Normal University, Haikou, China \\ Email: czchen@hainnu.edu.cn, yangsaisai1989@hotmail.com
}

Received 3 September 2014; revised 2 October 2014; accepted 13 October 2014

Academic Editor: Zechun Hu, Department of Mathematics, Nanjing University, China

Copyright @ 2014 by authors and Scientific Research Publishing Inc.

This work is licensed under the Creative Commons Attribution International License (CC BY).

http://creativecommons.org/licenses/by/4.0/

(c) $\underset{\mathrm{EY}}{\mathrm{EY}}$ Open Access

\section{Abstract}

Let $X$ be a Markov process, which is assumed to be associated with a (non-symmetric) Dirichlet form $(\mathcal{E}, D(\mathcal{E}))$ on $L^{2}(E ; m)$. For $u \in D(\mathcal{E})_{e}$, the extended Dirichlet space, we give necessary and sufficient conditions for a multiplicative functional to be a positive local martingale.

\section{Keywords}

\section{Markov Process, Dirichlet Form, Multiplicative Functional, Positive Local Martingale}

\section{Introduction}

Let $X=\left(\Omega, \mathcal{F},\left(\mathcal{F}_{t}\right)_{t \geq 0},\left(X_{t}\right)_{t \geq 0},\left(P_{x}\right)_{x \in E_{\Delta}}\right)$ be a (non-symmetric) Markov process on a metrizable Lusin space $E$ and $m$ be a $\sigma$-finite positive measure on its Borel $\sigma$-algebra $\mathcal{B}(E)$. Suppose that $(\mathcal{E}, D(\mathcal{E}))$ is a quasi-regular Dirichlet form on $L^{2}(E ; m)$ associated with Markov process $X$ (we refer the reader to [1] [2] for notations and terminologies of this paper). To simplify notation, we will denote by $u \in D(\mathcal{E})_{e}$ its $\mathcal{E}$-quasicontinuous $m$-version. If $u \in D(\mathcal{E})_{e}$, then there exist unique martingale additive functional (MAF in short) $M^{[u]}$ of finite energy and continuous additive functional (CAF in short) $N^{[u]}$ of zero energy such that

$$
u\left(X_{t}\right)-u\left(X_{0}\right)=M_{t}^{[u]}+N_{t}^{[u]}
$$

Let $\left(N(x, \mathrm{~d} y), H_{t}\right)$ be a Lévy system for $X$ and $v$ be the Revuz measure of the positive continuous additive functional (PCAF in short) $H$. For $t \geq 0$, we define the $[0, \infty]$-valued functional

$$
A_{t}^{u}=\int_{0}^{t}\left(\int_{E_{\partial}}\left(\mathrm{e}^{u(y)-u\left(X_{s}\right)}-1-\left(u(y)-u\left(X_{s}\right)\right)\right) N\left(X_{s}, \mathrm{~d} y\right)\right) \mathrm{d} H_{s}
$$


This paper is concerned with the following multiplicative functionals for $X$ :

$$
Z_{t}^{u}=\mathrm{e}^{M_{t}^{u}-\frac{1}{2}\left\langle M^{u, c}\right\rangle_{t}-A_{t}^{u}}
$$

where $\left\langle M^{u, c}\right\rangle_{t}$ is the sharp bracket PCAF of the continuous part $M^{u, c}$ of $M^{u}$.

In [3] under the assumption that $X$ is a diffusion process, then $Z_{t}^{u}=\mathrm{e}^{M_{t}^{u}-\frac{1}{2}\left\langle M^{u, c}\right\rangle_{t}}$ is a positive local martingale and hence a positive supermartingale. In [4], under the assumption that $u$ is bounded or $\mathrm{e}^{u} \in D(\mathcal{E})_{e}$, it is shown that $\left(Z_{t}^{u}, \mathcal{F}_{t}\right)_{t \geq 0}$ is a positive local martingale and hence induces another Markov process $Y$, which is called the Girsanov transformed process of $X$ (see [5]). Chen et al. in [5] give some necessary and sufficient conditions for $\left(Z_{t}^{u}, \mathcal{F}_{t}\right)$ to be a positive supermartingale when the Markov processes are symmetric. It is worthy to point out that the Beurling-Deny formula and Lyons-Zheng decomposition do not apply well to nonsymmetric Dirichlet forms setting. For the non-symmetric situations, $u \in D(\mathcal{E})_{e}$, an interesting and important question is that under what condition is $\left(Z_{t}^{u}, \mathcal{F}_{t}\right)_{t \geq 0}$ a positive local martingale?

In this paper, we will try to give a complete answer to this question when the Dirichlet forms are non-symmetric. We present necessary and sufficient conditions for $\left(Z_{t}^{u}, \mathcal{F}_{t}\right)_{t \geq 0}$ to be a positive local martingale.

\section{Main Result}

Recall that a positive measure $\mu$ on $(E, \mathcal{B}(E))$ is called smooth with respect to $(\mathcal{E}, D(\mathcal{E}))$ if $\mu(N)=0$ whenever $N \in B(\mathcal{E})$ is $\mathcal{E}$-exceptional and there exists an $\mathcal{E}$-nest $\left\{F_{n}\right\}_{n \geq 1}$ of compact subsets of $E$ such that

$$
\mu\left(F_{n}\right)<\infty \text { for all } k \geq 1
$$

Let $J(\mathrm{~d} x, \mathrm{~d} y)=\frac{1}{2} N(x, \mathrm{~d} y) v(\mathrm{~d} x), k(\mathrm{~d} x)=N(x, \partial) v(\mathrm{~d} x)$, We know from [6] that $J, k$ are Randon measures. Let $u \in D(\mathcal{E})_{e}, \quad Z_{t}^{u}$ be defined as in (1). Denote

$$
\begin{aligned}
& \mu_{u}(\mathrm{~d} x)=2 \int_{y \in E}\left(\mathrm{e}^{(u(y)-u(x))}-1-(u(y)-u(x))\right) J(\mathrm{~d} x, \mathrm{~d} y)+\left(\mathrm{e}^{-u(x)}-1+u(x)\right) k(\mathrm{~d} x), \\
& B_{t}^{u}=\sum_{s \leq t}\left[\mathrm{e}^{\left(u\left(X_{s}\right)-u\left(X_{s-}\right)\right)}-1-\left(u\left(X_{s}\right)-u\left(X_{s-}\right)\right)\right], \quad t \geq 0 .
\end{aligned}
$$

Now we can state the main result of this paper.

Theorem 1 The following are equivalent:

(i) $\left(Z_{t}^{u}, \mathcal{F}_{t}\right)_{t \geq 0}$ is a positive $P_{x}$-local martingale on $[0, \zeta)$ for q.e. $x \in E$.

(ii) $\left(B_{t}^{u}\right)_{t \geq 0}$ is locally $P_{x}$-integrable on $[0, \zeta)$ for q.e. $x \in E$.

(iii) $\mu_{u}$ is a smooth measure on $(E, \mathcal{B}(E))$.

Proof. (iii) $\Rightarrow$ (ii) Suppose that $\mu_{u}$ is a smooth measure on $(E, \mathcal{B}(E))$ and $\left\{F_{n}\right\}_{n \geq 1}$ is an $\mathcal{E}$-nest such that $\mu_{u}\left(F_{n}\right)<\infty$ and $I_{F_{n}} \cdot \mu_{u}$ is of finite energy integral for $n \geq 1$. Similar to Lemma 2.4 of [4],

$h^{t, n}:=E_{x}\left[\left(I_{F_{n}} \cdot A^{u}\right)_{t}\right]$ is quasi-continuous and hence q.e. finite. Denote $\tau_{n}:=\inf \left\{t>0 \mid X_{t} \notin F_{n}\right\}$. Then for $t \geq 0$,

$$
\begin{aligned}
E_{x}\left[B_{t \wedge \tau_{n}}^{u}\right] & \leq E_{x}\left\{\sum_{s \leq t} I_{F_{n}}\left(X_{s}\right)\left[\mathrm{e}^{u\left(X_{s}\right)-u\left(X_{s-}\right)}-1-\left(u\left(X_{s}\right)-u\left(X_{s-}\right)\right)\right]\right\} \\
& =E_{x}\left\{\int_{0}^{t} \int_{E_{\partial}} I_{F_{n}}\left(X_{s}\right)\left[\mathrm{e}^{u(y)-u\left(X_{s}\right)}-1-\left(u(y)-u\left(X_{s}\right)\right)\right] N\left(X_{s}, \mathrm{~d} y\right) \mathrm{d} H_{s}\right\} \\
& \leq E_{x}\left[\left(I_{F_{n}} \cdot A^{u}\right)_{t}\right]<\infty .
\end{aligned}
$$

Hence by proposition IV 5.30 of [1] $\left(B_{t}^{u}\right)_{t \geq 0}$ is locally $P_{x}$-integrable on $[0, \zeta)$ for q.e. $x \in E$.

(ii) $\Rightarrow$ (i) Assume that $\left(B_{t}^{u}\right)_{t \geq 0}$ is locally $P_{x}$-integrable on $[0, \zeta)$ for q.e. $x \in E$. One can check that for q.e. $x \in E$ the dual predictable projection of $\left(B_{t}^{u}\right)_{t \geq 0}$ on $[0, \zeta)$ is $A_{t}^{u}$. We set 


$$
\begin{aligned}
& B_{t}^{d}:=B_{t}^{u}-A_{t}^{u}, \\
& M_{t}:=M_{t}^{u}+B_{t}^{d} .
\end{aligned}
$$

Then $M_{t}$ is a local martingale on $[0, \zeta)$ and the solution $V_{t}^{u}$ of the stochastic differential equation (SDE)

$$
V_{t}^{u}=1+\int_{o}^{t} V_{s-}^{u} \mathrm{~d} M_{s}
$$

is a local martingale on $[0, \zeta)$. Moreover, by Doleans-Dade formula (cf. 9.39 of [7]), Note that $\left\langle M^{c}\right\rangle_{t}=\left\langle M^{u, c}\right\rangle_{t}$, we have that

$$
\begin{aligned}
V_{t}^{u} & =\exp \left\{M_{t}-\frac{1}{2}\left\langle M^{c}\right\rangle_{t} \prod_{s \leq t}\left(1+M_{s}-M_{s-}\right) \mathrm{e}^{M_{s-}-M_{s}}\right. \\
& =\exp \left\{M_{t}^{u}-\frac{1}{2}\left\langle M^{u, c}\right\rangle_{t}-A_{t}^{u}+B_{t}^{u}+\sum_{s \leq t}\left(u\left(X_{s}\right)-u\left(X_{s-}\right)+1-\mathrm{e}^{u\left(X_{s}\right)-u\left(X_{s-}\right)}\right)\right\} \\
& =\exp \left\{M_{t}^{u}-\frac{1}{2}\left\langle M^{u, c}\right\rangle_{t}-A_{t}^{u}\right\}=Z_{t}^{u} .
\end{aligned}
$$

So $\left(Z_{t}^{u}, \mathcal{F}_{t}\right)_{t \geq 0}$ is a $P_{x}$-local martingale.

Let $W_{t}:=\prod_{s \leq t}\left(1+M_{s}-M_{s-}\right) \mathrm{e}^{M_{s-}-M_{s}}$. Note that $M_{s}$ is a càdlàg process, there are at most countably many points at which $M_{s}-M_{s-} \neq 0$. Since by Lemma 7.27 of [7] $\sum_{s \leq t}\left(M_{s}-M_{s-}\right)^{2}<\infty \quad P_{x}$-a.e., there are only finitely many points $s$ at which $\left|M_{s}-M_{s-}\right|>1 / 2$, which give a finite non-zero contribution to the product. Using the inequality $|\ln (1+x)-x| \leq x^{2}$ when $|x| \leq 1 / 2$, we get

$$
\begin{aligned}
W_{t} & =\prod_{s \leq t:\left|M_{s}-M_{s-}\right| \leq 1 / 2}\left(1+M_{s}-M_{s-}\right) \mathrm{e}^{M_{s-}-M_{s}} \prod_{s \leq t: \mid M_{s}-M_{s-}>1 / 2}\left(1+M_{s}-M_{s-}\right) \mathrm{e}^{M_{s-}-M_{s}} \\
& \geq \prod_{s \leq t:\left|M_{s}-M_{s-}\right| \leq 1 / 2} \mathrm{e}^{-\left(M_{s}-M_{s-}\right) 2} \prod_{s \leq t: \mid M_{s}-M_{s-}>>1 / 2}\left(1+M_{s}-M_{s-}\right) \mathrm{e}^{M_{s-}-M_{s}} \\
& \geq \mathrm{e}^{-\sum_{s \leq t}^{\left(M_{s}-M_{s-}\right)^{2}}} \prod_{s \leq t: \mid M_{s}-M_{s-}>>1 / 2}\left(1+M_{s}-M_{s-}\right) \mathrm{e}^{M_{s-}-M_{s}}>0 .
\end{aligned}
$$

Therefore $\left(Z_{t}^{u}, \mathcal{F}_{t}\right)_{t \geq 0}$ is a positive $P_{x}$-local martingale on $[0, \zeta)$ for q.e. $x \in E$.

(i) $\Rightarrow$ (iii) Assume that $\left(Z_{t}^{u}, \mathcal{F}_{t}\right)_{t \geq 0}$ is a positive $P_{x}$-local martingale on $[0, \zeta)$ for q.e. $x \in E$, by Lemma 2.2 and Lemma 2.4 of [8],

$$
\begin{aligned}
L_{t} & :=\int_{0}^{t} \frac{1}{Z_{s-}^{u}} \mathrm{~d} Z_{t}^{u} \\
& =\ln Z_{t}^{u}-\ln Z_{0}^{u}+\frac{1}{2} \int_{0}^{t} \frac{1}{\left(Z_{s-}^{u}\right)^{2}} \mathrm{~d}\left\langle Z^{u, c}\right\rangle_{s}-\sum_{s \leq t}\left(\ln \frac{Z_{s}^{u}}{Z_{s-}^{u}}+1-\frac{Z_{s}^{u}}{Z_{s-}^{u}}\right) \\
& =M_{t}^{u}-\frac{1}{2}\left\langle M^{u, c}\right\rangle_{t}-A_{t}^{u}+\frac{1}{2} \int_{0}^{t} \frac{1}{\left(Z_{s-}^{u}\right)^{2}} \mathrm{~d}\left\langle Z^{u, c}\right\rangle_{s}+B_{t}^{u} .
\end{aligned}
$$

is a local martingale on $[0, \zeta)$. We set

$$
N_{t}:=L_{t}-M_{t}^{u}
$$

then $N_{t}=B_{t}^{u}-A_{t}^{u}-\frac{1}{2}\left\langle M^{u, c}\right\rangle_{t}+\frac{1}{2} \int_{0}^{t} \frac{1}{\left(Z_{s-}^{u}\right)^{2}} \mathrm{~d}\left\langle Z^{u, c}\right\rangle_{s}$ is also a local martingale on $[0, \zeta)$. Denote $N_{t}^{d}$ is the purely discontinuous part of $N_{t}$, by Theorem 7.17 of [7], there exist a locally bounded martingale $U_{t}$ and a 
local martingale of integrable variation $V_{t}$ such that $N_{t}^{d}=N_{0}^{d}+U_{t}+V_{t}$. Since $u$ is $E$-quasi-continuous, take an $\mathcal{E}$-nest $\left\{F_{n}\right\}_{n \geq 1}$ consisting of compact sets such that $\operatorname{Cap}\left(E \backslash F_{n}\right) \leq \frac{1}{3^{n+1}}$ and $\left.\tilde{u}\right|_{F_{n}}$ is continuous hence bounded q.e. for each $n \geq 1$. Denote

$$
\begin{aligned}
& D(\mathcal{E})_{F_{k}}:=\left\{f \in D(\mathcal{E}) \mid f=0 m-\text { a.e. on } E \backslash F_{k}\right\}, \\
& D(\mathcal{E})_{F_{k}, b}:=D(\mathcal{E})_{F_{k}} \cap \mathcal{B}(E)_{b} .
\end{aligned}
$$

Take a $\varphi \in L^{2}(E ; m), \quad 0<\varphi<1$. Set $h:=G_{1} \varphi$, where $\left(G_{\alpha}\right)_{\alpha>0}$ is the family of resolvents associated with $(\mathcal{E}, D(\mathcal{E}))$. Since $\bigcup_{n \geq 1} D(\mathcal{E})_{F_{n} . b}$ is dense in $D(\mathcal{E})$ w.r.t. the $\mathcal{E}_{1}^{1 / 2}$-norm, by proposition III. 3.5 and 3.6 of [1], there exists an $\mathcal{E}$-nest $\left\{F_{n}^{\prime}\right\}_{n \geq 1}$ consisting of compact sets and a sequence $\left\{f_{k}\right\}_{k \geq 1} \subset \bigcup_{n \geq 1} D(\mathcal{E})_{F_{n}, b}$ such that $\operatorname{Cap}\left(E \backslash F_{n}^{\prime}\right) \leq \frac{1}{3^{n+1}},\left.\widetilde{G_{1} \varphi}\right|_{F_{n}^{\prime}} \geq \delta_{n}$ for some $\delta_{n}>0$ and $\widetilde{f_{k}}$ converges to $\widetilde{G_{1} \varphi}$ uniformly on $F_{n}^{\prime}$ as $k \rightarrow \infty$ for each $n \geq 1$. Set $F_{n}^{\prime \prime}=F_{n} \bigcap F_{n}^{\prime}$. So there exists an non-negative $h_{n} \in \bigcup_{k \geq 1} D(\mathcal{E})_{F_{k}, b}$ and constant $a_{n}>0$ such that $h_{n} \geq a_{n}$ on $F_{n}^{\prime \prime}$. Suppose $h_{n} \in D(\mathcal{E})_{F_{k_{n}}, b}$, then

$$
\begin{aligned}
{\left[N^{d}, M^{h_{n}}\right]_{t} } & =\sum_{s \leq t}\left(N_{s}^{d}-N_{s-}^{d}\right)\left(M_{s}^{h_{n}}-M_{s-}^{h_{n}}\right) \\
& =\sum_{s \leq t}\left(U_{s}^{d}-U_{s-}^{d}\right)\left(M_{s}^{h_{n}}-M_{s-}^{h_{n}}\right)+\sum_{s \leq t}\left(V_{s}^{d}-V_{s-}^{d}\right)\left(h_{n}\left(X_{s}\right)-h_{n}\left(X_{s-}\right)\right) \\
& \leq\left[\sum_{s \leq t}\left(U_{s}-U_{s-}\right)^{2} \sum_{s \leq t}\left(M_{s}^{h_{n}}-M_{s-}^{h_{n}}\right)^{2}\right]^{1 / 2}+2\left\|h_{n}\right\|_{\infty} \sum_{s \leq t}\left|V_{s}-V_{s-}\right| .
\end{aligned}
$$

where $\|\cdot\|_{\infty}$ denotes the supremum norm. Recall that a locally bounded martingale $U_{t}$ is a locally square integrable martingales, $M_{t}^{h_{n}}$ is a locally square integrable martingales and $V_{t}$ is a local martingale of integrable variation. Therefore the quadratic variation $\left[N^{d}, M^{h_{n}}\right]$ is $P_{x}$-locally integrable for q.e.x $\in E$, hence there exist a predictable dual projection $\left\langle N^{d}, M^{h_{n}}\right\rangle_{t}$ which is a CAF of finite variation. Since

$$
\begin{aligned}
{\left[N^{d}, M^{h_{n}}\right]_{t} } & =\sum_{s \leq t}\left(B_{s}^{u}-B_{s-}^{u}\right)\left(M_{s}^{h_{n}}-M_{s-}^{h_{n}}\right) \\
& =\sum_{s \leq t}\left(\mathrm{e}^{u\left(X_{s}\right)-u\left(X_{s-}\right)}-1-\left(u\left(X_{s}\right)-u\left(X_{s-}\right)\right)\right)\left(h_{n}\left(X_{s}\right)-h_{n}\left(X_{s-}\right)\right) .
\end{aligned}
$$

the Revuz measure of $\left\langle N^{d}, M^{h_{n}}\right\rangle_{t}$ is

$$
\begin{aligned}
\mu_{\left\langle N^{d}, M^{h_{n}}\right\rangle}= & 2 \int_{\{y \in E: y \neq x\}}\left(h_{n}(y)-h_{n}(x)\right)\left(\mathrm{e}^{(u(y)-u(x))}-1-(u(y)-u(x))\right) J(\mathrm{~d} x, \mathrm{~d} y) \\
& +\left(\mathrm{e}^{-u(x)}-1+u(x)\right) k(\mathrm{~d} x) .
\end{aligned}
$$

Let $\left\{F_{k}^{\left.m{ }^{\prime \prime}\right\}_{k \geq 1}}\right.$ be a generalized $\mathcal{E}$-nest associated with $\mu_{\left\langle N^{d}, M^{h_{n}}\right\rangle}$ such that $\mu_{\left\langle N^{d}, M^{h_{n}}\right\rangle}\left(F_{k}^{\prime \prime \prime}\right)<\infty$ for each $k \geq 1$. Denote $D_{n}:=F_{n}^{\prime \prime} \cap F_{n}^{\prime \prime \prime}$, then $\operatorname{Cap}\left(E \backslash D_{n}\right) \leq \frac{1}{3^{n}}$ and $\left\{\bigcup_{k=1}^{n} D_{k}\right\}_{n \geq 1}$ is an $\mathcal{E}$-nest. Hence for any $g \in D(\mathcal{E})_{D_{n}, b}$, we have $\int_{E} g(x) \mathrm{d} \mu_{\left\langle N^{d}, M^{h_{n}}\right\rangle}<\infty$. On the other hand, as $\left.\tilde{u}\right|_{F_{n}}$ is bounded, there exists a positive constant $b_{n}$ such that $\mathrm{e}^{u(y)-u(x)}-1-\left.(u(y)-u(x))\right|_{D_{n} \times F_{k_{n}}}$ and $\mathrm{e}^{-u(x)}-1+\left.u(x)\right|_{D_{n} \bigcap_{F_{k_{n}}}}$ are not larger than $b_{n}$. Because $J(\mathrm{~d} x, \mathrm{~d} y), k(\mathrm{~d} x)$ are Radon measure and $h_{n}, g$ are bounded, 


$$
\begin{aligned}
& \int_{E \times E \backslash d} g(x) h_{n}(x)\left(\mathrm{e}^{u(y)-u(x)}-1-(u(y)-u(x))\right) J(\mathrm{~d} x, \mathrm{~d} y) \\
& =\int_{E \times E \backslash d} g(x) h_{n}(\mathrm{y})\left(\mathrm{e}^{u(y)-u(x)}-1-(u(y)-u(x))\right) J(\mathrm{~d} x, \mathrm{~d} y) \\
& \quad-\int_{E \times E \backslash d} g(x)\left(h_{n}(y)-h_{n}(x)\right)\left(\mathrm{e}^{u(y)-u(x)}-1-(u(y)-u(x))\right) J(\mathrm{~d} x, \mathrm{~d} y) \\
& =\int_{E \times E \backslash d} g(x) h_{n}(y)\left(\mathrm{e}^{u(y)-u(x)}-1-(u(y)-u(x))\right) J(\mathrm{~d} x, \mathrm{~d} y) \\
& \quad+\frac{1}{2} \int_{E} h_{n}(x) g(x)\left(\mathrm{e}^{-u(x)}-1+u(x)\right) k(\mathrm{~d} x)-\frac{1}{2} \int_{E} g(x) \mathrm{d} \mu_{\left\langle N^{d}, M^{h_{n}}\right\rangle} \\
& =\int_{D_{n} \times F_{k_{n}} \backslash d} g(x) h_{n}(y)\left(\mathrm{e}^{u(y)-u(x)}-1-(u(y)-u(x))\right) J(\mathrm{~d} x, \mathrm{~d} y) \\
& \quad+\frac{1}{2} \int_{D_{n}} \bigcap_{F_{k_{n}}} h_{n}(x) g(x)\left(\mathrm{e}^{-u(x)}-1+u(x)\right) k(\mathrm{~d} x)-\frac{1}{2} \int_{E} g(x) \mathrm{d} \mu_{\left\langle N^{d}, M^{h_{n}}\right\rangle}<\infty
\end{aligned}
$$

As inequality $\mathrm{e}^{u(y)-u(x)}-1-(u(y)-u(x)) \geq 0$ on $E$ and $h_{n} \geq a_{n}$ on $F_{n}^{\prime \prime}$, we have for any non-negative $f \in D(\mathcal{E})_{D_{n}, b}$,

$$
\begin{aligned}
& \int_{E} f(x) \mu_{u}(\mathrm{~d} x) \\
& =2 \int_{E \times E \backslash d} f(x)\left(\mathrm{e}^{u(y)-u(x)}-1-(u(y)-u(x))\right) J(\mathrm{~d} x, \mathrm{~d} y)+\int_{E} f(x)\left(\mathrm{e}^{-u(x)}-1+u(x)\right) k(\mathrm{~d} x) \\
& \leq 2\left(a_{n}\right)^{-1} \int_{D_{n} \times E \backslash d} h_{n}(x) f(x)\left(\mathrm{e}^{u(y)-u(x)}-1-(u(y)-u(x))\right) J(\mathrm{~d} x, \mathrm{~d} y)+\int_{D_{n}} f(x)\left(\mathrm{e}^{-u(x)}-1+u(x)\right) k(\mathrm{~d} x)<\infty .
\end{aligned}
$$

For $\left\{\bigcup_{k=1}^{n} D_{k}\right\}_{n \geq 1}$ is an $\mathcal{E}$-nest consisting of compact sets, similar to $h_{n}$, we can construct an $\mathcal{E}$-nest $\left\{\bigcup_{k=1}^{n} D_{k}^{\prime}\right\}$ consisting of compact sets such that $D_{n}^{\prime} \subset D_{n}$ for each $n \geq 1$. And there exists a sequence nonnegative $\left\{h_{n}^{\prime}\right\}_{n \geq 1} \subset \bigcup_{k \geq 1} D(\mathcal{E})_{D_{k}, b}$ such that $h_{n}^{\prime} \geq c_{n}$ on $D_{n}^{\prime}$ for each $n \geq 1$ and some positive $c_{n}>0$. Since $\mu_{u}\left(\bigcup_{k=1}^{n} D_{k}^{\prime}\right) \leq \sum_{k=1}^{k=n} c_{k}^{-1} \int_{E}\left(h_{k^{\prime}}(x)\right) \mu_{u}(\mathrm{~d} x)<\infty, \mu_{u}$ is a smooth measure on $(E, \mathcal{B}(E))$.

\section{Acknowledgments}

We are grateful to the support of NSFC (Grant No. 10961012).

\section{References}

[1] Ma, Z.M. and Rockner, M. (1992) Introduction to Theory of (Non-Symmetric) Dirichlet Forms. Springer-Verlag, Berlin. http://dx.doi.org/10.1007/978-3-642-77739-4

[2] Fukushima, M., Oshima, Y. and Takeda, M. (1994) Dirichlet Forms and Symmetric Markov Processes. Walter de Gruyter Berlin, New York. http://dx.doi.org/10.1515/9783110889741

[3] Chen, C.-Z. and Sun, W. (2009) Girsanov Transformations for Non-symmetric Diffusions. Canadian Journal of Mathematics, 61, 534-547. http://dx.doi.org/10.4153/CJM-2009-028-7

[4] Chen, Z.-Q. and Zhang, T.-S. (2002) Girsanov and Feynman-Kac Type Transformations for Symmetric Markov Processes. Annales de l'Institut Henri Poincare (B) Probability and Statistics, 38, 475-450. http://dx.doi.org/10.1016/S0246-0203(01)01086-X

[5] Chen, C.-Z., Ma, Z.-M. and Sun, W. (2007) On Girsanov and Generalized Feynman-Kac Transformations for Symmetric Markov Process. World Scientific, 10, 141-163.

[6] Oshima. Y. (2013) Semi-Dirichlet Forms and Markov Processes. Walter de Gruyter, Berlin. http://dx.doi.org/10.1515/9783110302066

[7] He, S.W., Wang, J.G. and Yan, J.A. (1992) Semimartingale Theory and Stochastic Calculus. Science Press, Beijing.

[8] Kallsen, J. and Shiryaev, A.N. (2002) The Cumulant Process and Esscher's Change of Measure. Finance Stochast, 6, 397-428. http://dx.doi.org/10.1007/s007800200069 
Scientific Research Publishing (SCIRP) is one of the largest Open Access journal publishers. It is currently publishing more than 200 open access, online, peer-reviewed journals covering a wide range of academic disciplines. SCIRP serves the worldwide academic communities and contributes to the progress and application of science with its publication.

Other selected journals from SCIRP are listed as below. Submit your manuscript to us via either submit@scirp.org or Online Submission Portal.
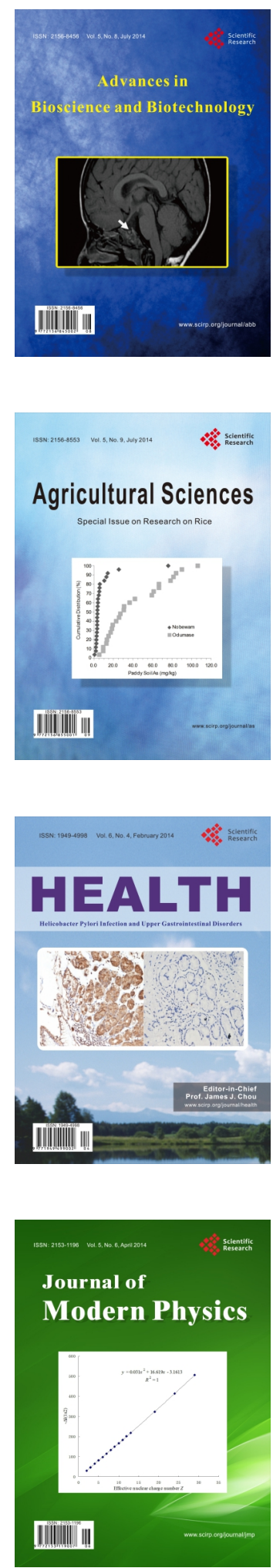
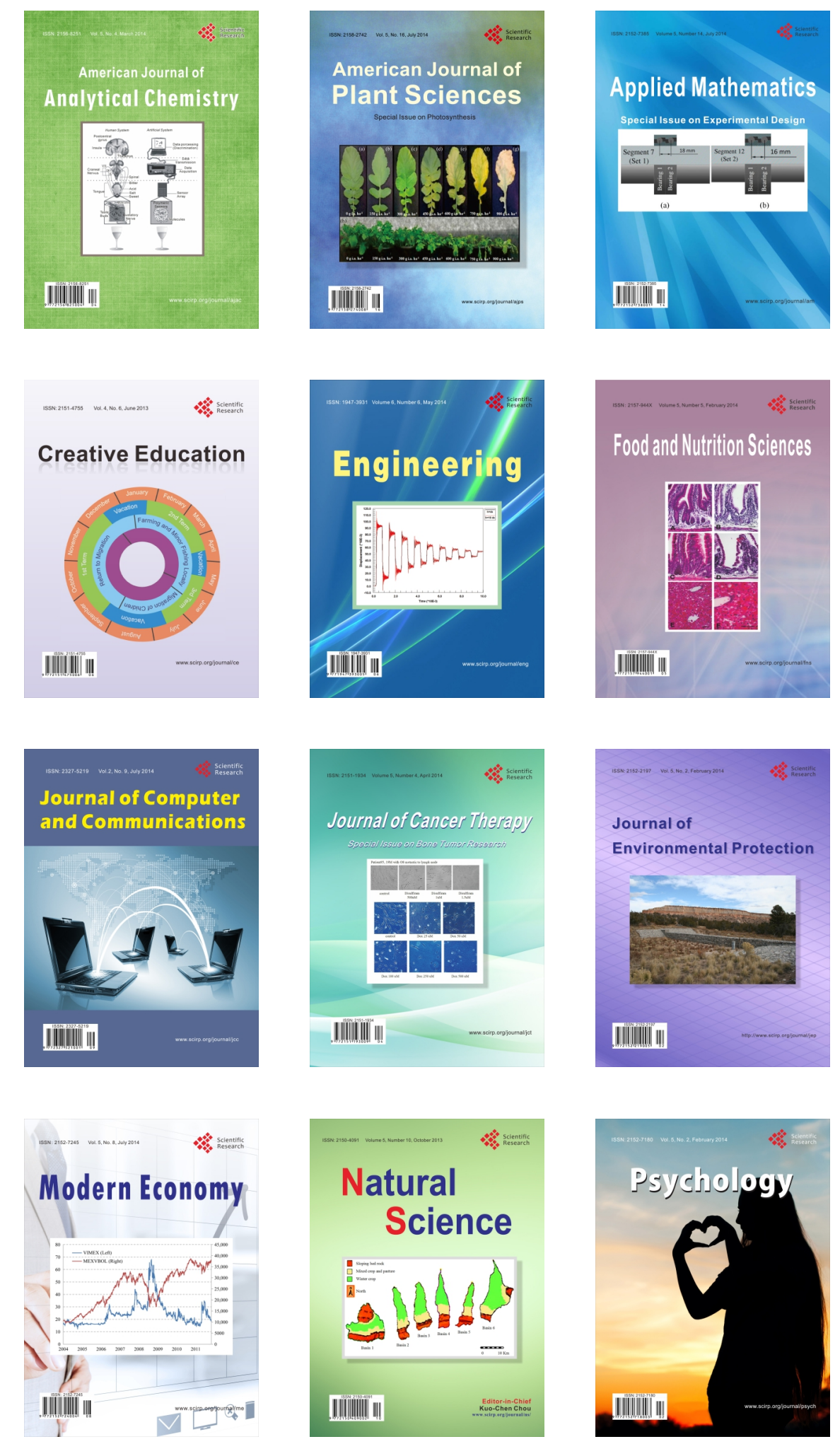